\title{
Universidad Nacional Agraria y su responsabilidad en la conservación y manejo de recursos naturales: caso Isla Zapatera
}

\section{Universidad Nacional Agraria and its responsibility in the conservation and management of natural resources: zapatera island case}

\author{
Emilio Pérez-Castellón' ${ }^{1}$ Nadarajah Sriskandarajah ${ }^{2}$, Alex Arévalo-Vásquez ${ }^{3}$ \\ ${ }^{1}$ Dr. Profesor Universidad Nacional Agraria, Nicaragua, emiliopc21@hotmail.com, ${ }^{2}$ Dr. Profesor Universidad Agrícola de \\ Suecia, Nadarajah.Sriskandarajah@slu.su, ${ }^{3}$ M.Sc. Oficial de Proyectos, Svenska Naturskyddsföreningen, Suecia, arevalito@ \\ gmail.com
}

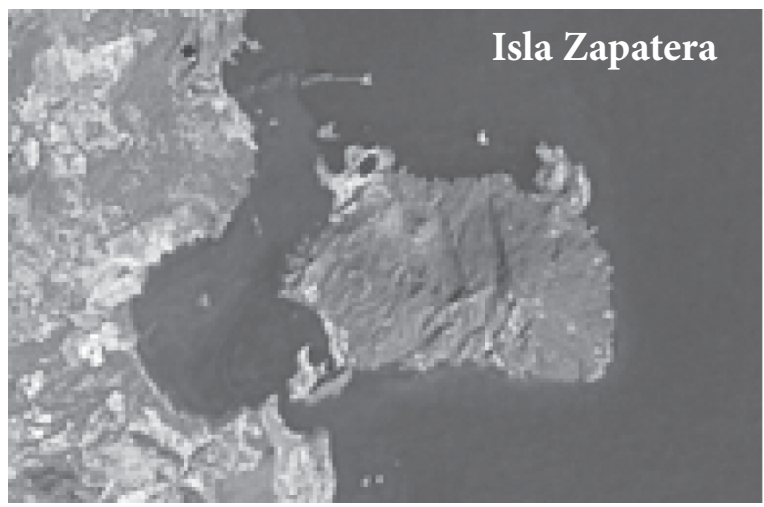

\section{RESUMEN}

El pensamiento sistémico es un método holístico, incluido en el conjunto de métodos conocidos como sistemas blandos. Los conceptos de conservación y manejo de los recursos naturales y vida comunitaria, se convierten en un dilema de difícil concertación en la realidad. Actualmente, es necesario encarar situaciones complejas y dinámicas en el quehacer agropecuario y el manejo de los recursos naturales y el ambiente. Los beneficiarios directos son pobladores y propietarios de áreas protegidas; indirectos, actores externos representados por organizaciones gubernamentales y no gubernamentales, incluyendo centros de educación superior e investigación. Utilizando sistemas blandos y pensamiento sistémico, aprendizaje experiencial y ciclo de Kolb, así como mapas mentales, imagen enriquecida y diferentes herramientas participativas, se inició un proceso en la Isla Zapatera, para generar un intercambio de visiones y pareceres y una plataforma de comunicación e interacción que acerque las posiciones comunes de los diferentes actores y permita avanzar hacia la actualización y definición conjunta de un plan de manejo integral y consensuado del área protegida, Parque Nacional Archipiélago Isla Zapatera. Los resultados son halagadores en el sentido de que se logró establecer tal plataforma, y el grado de comunicación e intercambio, es clave en el logro de posiciones ventajosas para los diferentes actores, especialmente a los habitantes del Archipiélago. Este trabajo fur coordinado por la Universidad Nacional Agraria (Nicaragua) y la Universidad Agrícola de Suecia, apoyados por el Ministerio de Recursos Naturales y del Ambiente, y otros organismos gubernamentales y no gubernamentales.

Palabras clave: pensamiento sistémico, áreas protegidas, Nicaragua, comunicación ambiental, investigación acción.
ABSTRACT

Thinking systems are a holistic method, included in the set of methods known as soft systems. The concepts of conservation and natural resource management and community life, become a dilemma of difficult conciliation in reality. Currently, it is necessary to deal with complex and dynamic situations in the daily agricultural and natural resource management and environment. The direct beneficiaries are residents and owners of protected areas; indirect external actors represented by organizations, governmental and non-governmental organizations, including institutions of higher education and research. Using soft systems and thinking systems, experiential learning and Kolb cycle as well as mind maps, rich picture and various participatory tools, we began a process in Zapatera Island, to generate an exchange of views and opinions and a platform for communication and interaction that brings the common positions of the different actors and enable progress towards updating and joint identification of a comprehensive management and consensual plan for the protected area, Zapatera Island Archipelago National Park. The results are flattering in the sense that they were able to establish such a platform and the degree of communication and exchange, is key in achieving advantageous positions for different actors especially the inhabitants of the archipelago. Coordinate UNA and SLU, supported by MARENA, and other NGOs and GOs.

Keyword: System thinking, protected areas, Nicaragua, environmental communication, action research.

Recibido: 18 de marzo 2015

Aceptado: 26 de agosto 2015 


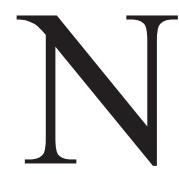

icaragua es el segundo país más empobrecido del hemisferio occidental después de Haití, y enfrenta muchos retos de carácter socioeconómico. La mayoría de la población más empobrecida, vive en las áreas rurales y son el sector más vulnerable a los fenómenos naturales tales como sequías, inundaciones, incendios, erupciones volcánicas y terremotos. Su extensión es estimada en $130300 \mathrm{~km}^{2}$, que convierte al país en el más grande de Centroamérica, con una población estimada de 5142098 habitantes según el censo poblacional del año 2005 (U.S. Department of State, 2013; Infoplease, 2013; Universia, 2013; INIDE-INEC, 2013; Arévalo, 2010).

Para el año 1983 se declaró a la Isla Zapatera y varias isletas que se encuentran a su alrededor, como Parque Nacional Archipiélago Isla Zapatera (PNAIZ). En ese entonces se pensó que era necesario proteger la enorme riqueza arqueológica del archipiélago, ya que aparentemente la isla principal y sus isletas se usaron como centros funerarios y ceremoniales, así como de capacitación nativa, más que para asentamientos permanentes. Adicionalmente, se consideró de mucha importancia por su representatividad y escasez, su Flora y Fauna, tanto terrestre como acuática. Se estima que la extensión del parque es de $52 \mathrm{~km}^{2}$ y la Isla Zapatera es la segunda más grande dentro del Lago Cocibolca, después de la Isla Ometepe (MARENA, 2013). Desde el año 1983 existe un Plan de Manejo que está vigente.

El PNAIZ, está categorizado como un área protegida de clase II, para el caso de esta clase las únicas actividades permitidas consisten en investigaciones y estudios, educación ambiental e interpretación, turismo sostenible y la recreación. También se establece que el único tipo de infraestructura que se puede tolerar es la que está definida para la promoción, seguimiento y control del parque a través del Ministerio del Ambiente y los Recursos Naturales (MARENA) y por ende no deben de existir asentamientos con pobladores. Por supuesto que toda actividad de pesca, caza y aprovechamiento de los productos forestales maderables y no maderables también son ilegales, sin embargo, todas estas regulaciones y prohibiciones no están siendo efectivas ni controladas por el MARENA, dadas sus limitaciones y su poca capacidad de cobertura en términos de recursos humanos, materiales y financieros en el propio sitio del PNAIZ, para asegurar la correcta aplicación de todas las regulaciones (MARENA, 2013; Arévalo, 2010).

La Universidad Nacional Agraria (UNA) para cumplir con su misión, que claramente establece su “....Compromiso Social Universitario...." y la Universidad Agrícola de Suecia (SLU), unieron esfuerzos para explorar la posibilidad de desarrollar un proceso que permitiera alcanzar una comprensión holística de la vida de los habitantes, así como la identificación de los principales problemas en relación al uso y manejo de los recursos naturales, y a la vez proponer formas de comunicación, integración, intercambio $\mathrm{y}$ acciones que eventualmente permitan alcanzar un mejor bienestar de los habitantes de las comunidades y se mejore el manejo de los recursos naturales en el Parque Nacional Archipiélago Isla Zapatera. Todo el proceso está basado en el uso de metodologías participativas y sistémicas.

El dilema de la conservación y el uso y manejo adecuado de los recursos naturales, en contraposición al desarrollo y bienestar social, de las comunidades que viven gracias al uso de esos recursos naturales, ha sido de difícil concertación y solución, especialmente cuando se trata de comunidades que se encuentran insertas y/o cercanas a las áreas protegidas. Esto es debido, en parte, a que los habitantes de tales comunidades, en el afán de los Gobiernos y Ministerios de los recursos naturales y del ambiente, en lugar de proteger y conservar los recursos naturales y el ambiente en ciertos lugares de interés, generalmente son dejados fuera de cualquier decisión y/o planificación que implique regulaciones y controles del uso que tradicionalmente han realizado sobre estos recursos naturales para su sobrevivencia y desarrollo.

Esta forma de encarar esta situación, más bien ha generado un efecto contrario al deseado por las instancias gubernamentales, dado que la conservación y protección de los recursos naturales y el ambiente se ha visto afectada y en algunos casos disminuida. Sin embargo, existen algunas experiencias en donde la participación genuina de las comunidades, ha permitido alcanzar avances significativos en el manejo conjunto y colaborativo de los recursos de las áreas protegidas, lo que ha conducido a un beneficio recíproco para los gobiernos, las comunidades y la biodiversidad.

Adicionalmente, existe una seria complicación en el caso de la tenencia de la tierra, ya que hay una incertidumbre acerca de la veracidad de los aseguramientos de los diferentes actores, tales como los pobladores, los propietarios grandes y el gobierno a través del MARENA, quienes plantean diferentes versiones en relación a quién posee o tiene títulos de propiedad y cuánta área poseen (MARENA, 2013; Arévalo, 2010).

La situación es compleja, dado que a pesar de las prohibiciones, se continúa con la pesca, caza y tala ilegal, además del saqueo y contrabando de evidencias arqueológicas (MARENA, 2013; Arévalo, 2010). Se necesita de acciones urgentes y por tal razón la UNA y SLU decidieron colaborar en la búsqueda de alternativas para el mejoramiento de la vida de los isleños y la conservación y protección de la biodiversidad, a través del uso de los sistemas de investigación blandos, fundamentados en metodologías participativas. La actualización del Plan de Manejo con un enfoque holístico, integral y participativo es de suma importancia para mejorar la situación.

\section{METODOLOGÍA}

La metodología de los sistemas blandos (MSB) se usó en conjunto con los principios de la investigación acción participativa (IAP) y los diferentes instrumentos $\mathrm{y}$ herramientas que proporciona el diagnóstico rural participativo (DRP). 
Los sistemas blandos, además de constituirse en un proceso ordenado, permiten la flexibilidad para abordar situaciones en las cuales el comportamiento o acciones y las percepciones humanas pareciesen ser los factores dominantes donde las metas, objetivos e incluso las interpretaciones de los eventos son todas de carácter problemáticas. La MSB permite que sus principios puedan ser adoptados y adaptados, para su uso en una situación real, en la cual la gente quiere intentar la toma de acciones para mejorar tal situación (Checkland y Poulter, 2006; Naughton, 1984).

Según Naughton (1984), la MSB se basa en las siguientes ideas claves: 1) Los problemas son constructos de mentes preocupadas, 2) La gente maneja diferentes apreciaciones de las situaciones, porque realmente las miran de distintas maneras, debido a la existencia de diferentes cosmovisiones, 3) Las eventuales alternativas de solución también son constructos intelectuales, 4) Las cosmovisiones conflictivas originan desacuerdos en relación a la descripción del real estado de la situación, así como de lo que podría ser la situación deseada, 5) En la vida real, cada problema interactúa con otros problemas lo que se convierte en un desorden, 6) Los facilitadores de la MSB, ayudan a la gente a comprometerse en una situación problemática a través del análisis, debate serie flexible de ciclos, en la forma de pasos holísticos y no separados (Pérez, 2000; Pretty, 1998; Chambers, 1997).

El método convencional de arriba hacia abajo (topdown), presenta limitaciones en el logro de los esperados resultados exitosos en el desarrollo real de las áreas rurales especialmente para pequeños campesinos con muy limitados recursos, la razón de usar los principios de la IAP, es debido a que actualmente existen muchas experiencias, estudios e información que han demostrado que esta metodología proporciona la oportunidad de poner a los miembros de las comunidades primero y que los investigadores externos jueguen un papel de facilitadores, más que de decisores (Pérez, 2000; Pretty, 1998; Chambers, 1997).

Herramientas del trabajo de campo. De acuerdo a las enseñanzas y principios de la metodología definida en este

Observación participante Transectos

Entrevistas semiestructuradas Líneas de tiempo

Diagrama de Venn

Mapeo comunitario

Mapeo mental

Matrices
Reuniones Comunitarias

Talleres

Imagen enriquecida (figura 1) Grupos de trabajo y generar propuestas de cambios fructíferos, 7) El facilitador no puede estar divorciado o desligado del análisis, el facilitador no solo intenta comprender la situación, sino también ayudar a cambiarla para mejorar.

Los esfuerzos que se realizan a nivel internacional en el ámbito del desarrollo de las áreas rurales y la degradación del ambiente y de los recursos naturales, han identificado de manera creciente la necesidad de emplear métodos más innovadores. Estos métodos tienen que usarse con un enfoque holístico, en los diversos contextos ecológicos y sociales, y enfatizar la participación real y significativa de los pobladores locales en la planificación y

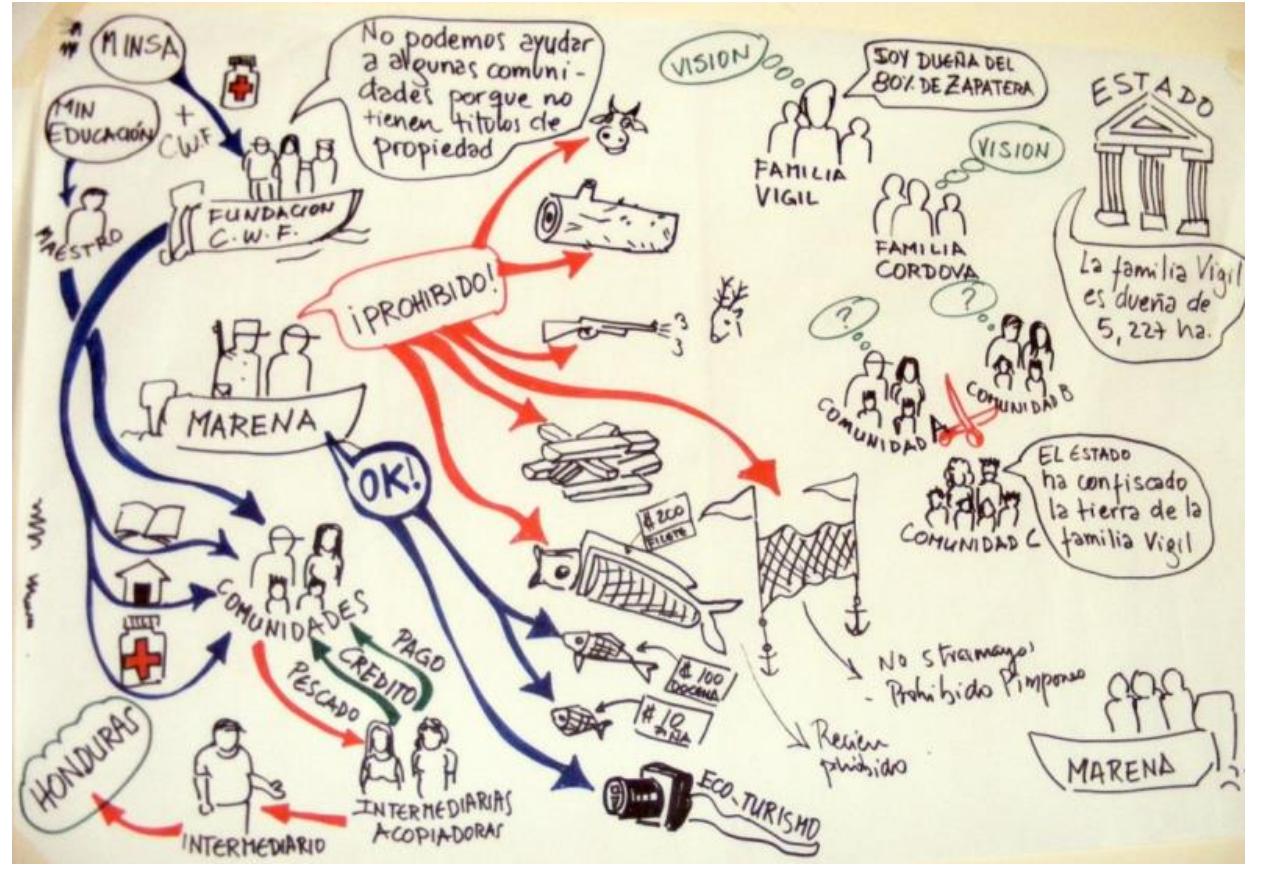

Figura 1. Imagen enriquecida de la situación en la Isla Zapatera. Fuente: Arévalo, (2010). ejecución de las acciones. De esta manera la IAP es un método para abordar situaciones complejas. A través de este método, que incluye la acción reflexiva, se busca el mejoramiento y la construcción de conocimiento nuevo. De esta manera se combinan la acción y su reflexión, así como la teoría y la práctica con la mayor participación. En el proceso de IAP, la integración de la investigación y la acción se efectúa a través de una estudio en el PNAIZ, se pusieron en práctica diferentes métodos y herramientas para poder conocer a fondo y atender la problemática planteada en el Parque. Entre los métodos y herramientas se incluyeron:

En la realización de todas estas actividades, se fue ampliando la participación de solo los facilitadores de la UNA con la SLU y pobladores de la comunidad, que ocurrió 
en un principio, hacia otros actores relevantes en el quehacer, manejo y uso del PNAIZ en la medida que se iba dominando mejor la dimensión de la problemática, que definitivamente es muy compleja y necesita del concurso de muchos actores e instancias tanto gubernamentales como no gubernamentales.

\section{RESULTADOS}

Hasta el momento se han obtenido resultados producto de dos etapas iniciales de involucramiento por parte de la UNA y SLU. La primera sucedió en el 2009-2010 a través de la realización de una tesis de maestría de un estudiante de Suecia y la segunda etapa en el período 2012-2013 a través de un proyecto pequeño de seguimiento financiado por el Consejo Sueco de Investigación.

En la primera etapa (2009-2010), se realizó un primer estudio con el objetivo de de comprender la relación entre las formas o medios de vida de los pobladores en dos, de un total de siete comunidades, y su relación con el manejo y uso de los recursos naturales. Entre los resultados se logró conocer que la riqueza y acceso de los pobladores a capital y recursos, es muy diferente entre las familias de las comunidades y entre las dos comunidades. Con relación al acceso a la tierra, también se encontró que una de las comunidades tiene más acceso a la tierra que la otra y que en el cercano futuro, se generarán conflictos al crecer la población de ambas comunidades. Otro descubrimiento es que al tratarse de un Parque Nacional, MARENA regula y prohíbe, incluso que no existan habitantes en el Archipiélago, sin embargo, se realizan muchas acciones ilegales, tanto por habitantes como gentes externas al área, por supuesto esta situación es fuente de muchos conflictos. La situación de acceso al mercado para comercializar sus pocos productos, también se constituye en una dificultad, que les hace presionar más a los recursos naturales. El mismo carácter de Parque Nacional impide que organizaciones gubernamentales y no gubernamentales tengan presencia, con diferentes tipos de programas socio productivos, lo que hace difícil la atención en muchos servicios básicos para la población. Este trabajó concluyó que la gestión de los recursos naturales en la Isla de Zapatera se puede mejorar al abordar primero la cuestión de la pobreza en las comunidades que habitan la isla; promoviendo el diálogo y el debate entre las diferentes partes interesadas; $\mathrm{y}$ con una mayor cooperación en investigación entre las comunidades locales, el ministerio del ambiente, autoridades, profesionales de campo, investigadores y donantes.

En la segunda etapa (2012-2013), el principal objetivo del estudio, además de darle continuidad al proceso, de acuerdo a la conclusión, se concentró en llegar a un nivel de diálogo, construcción e intercambio de conocimiento y empoderamiento de los actores del Archipiélago, a través de plataformas de comunicación que la UNA y SLU, promovieron con otros actores externos al Archipiélago. Esta segunda etapa se inició con una visita al PNAIZ y un taller para realizar un análisis comparativo en relación a la información generada. La situación de los pobladores en relación al uso y manejo de los recursos naturales no varió mucho, más se puede inferir que más bien se deterioró, porque la pesca disminuyó se encareció el producto y la presión sobre el recurso fue mayor y siguió el círculo vicioso de la degradación. Algo similar estaba sucediendo con la leña y la madera. Sin embrago, los pobladores y el MARENA coincidieron en que sus relaciones mejoraron y ahora el MARENA estaba más flexible, pues les permitía cuotas de leña y madera. Se pudo explorar la visión e ideas de acción de los pobladores y se pudo apreciar la existencia de muchos deseos de superación y actividades basadas en la apreciación de la naturaleza y el desarrollo eco turístico. Mencionaron estar conscientes de la necesidad de capacitarse en actividades menos destructivas del ambiente y en recibir apoyo de entidades gubernamentales y no gubernamentales, que les permita alcanzar mejores niveles de vida, pero con la compañía de instituciones como la UNA y SLU. Otro momento implicó una reunión-taller con el personal de base de MARENA que están a cargo del Sistema Nacional de Áreas Protegidas (SINAP) y por ende del PNAIZ. Durante este evento se abordaron aspectos como línea de tiempo del PNAIZ, visión de futuro, barreras-obstáculos y un plan de acción. Los resultados indicaron una coincidencia de la necesidad de mantener la comunicación, de realizar trabajos conjuntos y agilizar el proceso de actualización del Plan de manejo del PNAIZ, además de la problemática general manifestada por los pobladores y algunos otros actores externos. Valoraron como muy positiva la incidencia de la UNA y SLU en todo el proceso de búsqueda de acciones encaminadas a brindarle respuestas a tal problemática. Finalmente se realizó un taller en la ciudad de Granada, al cual asistieron más de 40 personas representantes de las siete comunidades del PNAIZ, más de 19 instituciones gubernamentales $\mathrm{y}$ no gubernamentales, privadas $\mathrm{y}$ universidades nacionales e internacionales. En este taller se obtuvieron importantes insumos e intercambios de un amplio escenario de actores interesados en el desarrollo del PNAIZ, se abordaron aspectos relacionados al plan de manejo, medios de vida alternativos, intercambio de información, organización, cooperación entre comunidades-gobierno, estación de investigación, educación y tenencia de la tierra.

Adicionalmente, incluso existió la oportunidad de poder desarrollar un curso corto internacional de $\mathrm{PhD}$, organizado entre SLU y la UNA, para abordar el uso de métodos para la indagación en situaciones conflictivas reales. El curso se denominó: conservación, desarrollo y comunicación en diversos paisajes: teoría y métodos. Toda la parte práctica se desarrollo en el PNAIZ y se cerró con un foro en donde participaron los estudiantes de doctorado originarios de América, Asia, África y Europa, pobladores, representantes de instituciones gubernamentales y las máximas autoridades de la UNA, así como los facilitadores de ambas universidades. 


\section{CONCLUSIONES}

A través de este estudio, la UNA está asumiendo y cumpliendo la declaración de su Misión, además de sus principios y valores, como es el compromiso social universitario, de cara a las necesidades del país. También SLU, se plantea que la esencia de la investigación y la enseñanza es el estudio multidisciplinar. Así que una conclusión tácita de este trabajo es el establecimiento de una alianza interuniversitaria que ha generado un proceso real para la sociedad.

Con la visión de desarrollar el proceso, en vez de un proyecto más, en la búsqueda de resolución a los conflictos que se generan por el desarrollo humano en contraposición a la conservación de la biodiversidad, podemos asegurar que ambas universidades han sido capaces de contribuir con el desarrollo del conocimiento necesario que ha permitido ir avanzando, paulatina pero inexorablemente, en base a, los lineamientos y enseñanzas mencionadas por los diferentes autores en términos de la aplicación en una situación real, para promover su cambio, de la MSB, IAP y el DRP.

Una vez establecida y analizada la situación real de los pobladores y el manejo de los recursos naturales del PNAIZ, se comprobó que la MSB, IAP y el DRP lograron generar espacios y plataformas de comunicación, intercambio de puntos de vista, visiones, conciliaciones, incluso hasta negociaciones en el ámbito del uso y manejo de los recursos naturales en relación al bienestar y mejora de la vida de los pobladores.
Las instituciones nacionales e internacionales tuvieron la oportunidad de analizar la situación real del PNAIZ y contribuir a través de la facilitación, en la búsqueda de resolución a los conflictos y avanzar en términos de sugerir planes de acción que permitan mejorar algunas situaciones que se pueden abordar a través de la actualización del plan de manejo del Parque Nacional Archipiélago Isla Zapatera.

La UNA está promoviendo en el MARENA, la mencionada actualización, ya que MARENA ha logrado sensibilizarse y conocer de primera mano que las regulaciones y prohibiciones no están logrando las metas de conservación de la biodiversidad, deseadas, sino por el contrario, la biodiversidad se ve deteriorada aún más.

Como resultado del foro realizado en el PNAIZ, al final de curso de doctorado, se organizó una comisión interinstitucional de organismos nacionales y la UNA quedó como integrante de esta comisión. El objetivo de la comisión es abordar la problemática, desde los diferentes ángulos, para ir incidiendo en la mejora del bienestar. Finalmente, los estudiantes de doctorado presentes en el curso de PhD, concluyeron que la combinación de la teoría combinada con la práctica en el Parque Nacional, les fue de significativa importancia en el diseño, elaboración y planeamiento de sus propias investigaciones y estudios de sus disertaciones doctorales.

\section{REFERENCIAS BIBLIOGRÁFICAS}

Arévalo, AR. 2010. Enhancing Natural Resources Management and Livelihoods in Zapatera Archipelago National Park, Nicaragua. An Action Research Study with Residents of two Communities in Zapatera Island. Masters Thesis. Swedish University of Agricultural Sciences. Uppsala, Sweden. 49 p.

Chambers, R. 1997. Whose Reality Counts? Putting the First Last. Intermediate Technology Publications, London, UK. 297 p.

Checkland, P; Poulter, J. 2006. Learning for Action: A Short Definitive Account of Soft Systems Methodology and its use for Practitioners, Teachers and Students. Antony Rowe Ltd. UK. 200 p.

Infoplease. 2013. Nicaragua. (en línea). Consultado 31 oct. 2013. Disponible en: http://www.infoplease.com/country/nicaragua.html

INIDE; INEC. (Instituto Nacional de Información de Desarrollo, NI); (Instituto Nacional de Estadísticas y Censo, NI). 2013. VIII Censo de Población y IV de vivienda, 2005. Nicaragua. (en línea). Consultado 31 oct. 2013. Disponible en: http://www.inide. gob.ni/censos2005/ResumenCensal/Resumen2.pdf

MARENA (Ministerio del Ambiente y los Recursos Naturales, NI) 2013. Reserva Natural Isla Zapatera. (en línea). Consultado 31 oct. 2013. Disponible en: http://www.marena.gob.ni/index.php?option=com content\&task=view\&id=218\&Itemid=535

Naughton, J. 1984. Soft Systems Analysis: An Introductory Guide. The Open University Press.

Pérez, E. 2000. Empowerment in Pacora: Use of Local Knowledge and Participatory Action Research to Promote Sustainable Agriculture and Natural Resources Management. Ph.D. Thesis. Cornell University. Ithaca, NY. 157 p.

Pretty, J. 1998. The living Land: Agriculture, Food and Community Regeneration in Rural Europe. Earthscan Pub. Ltd. London, UK. 324 p.

Universia. 2013. Estudios Internacionales. Estudios de Latinoamérica. Población de Nicaragua. (en línea). Consultado 31 oct. 2013. Disponible en: http://internacional.universia.net/latinoamerica/datos-paises/nicaragua/poblacion.htm

U.S. Department of State. 2013. Nicaragua Country Specific Information. (en línea). Consultado 31 oct. 2013. Disponible en: http:// travel.state.gov/travel/cis_pa_tw/cis/cis_985.html 